\title{
Suspected Respiratory Depression Associated with Use of a Transdermal Fentanyl Patch
}

\section{TO THE EDITOR:}

Transdermal fentanyl has been proved as being effective in the long-term management of chronic noncancer pain $(1,2)$. Besides its efficacy, the transdermal route is also safe, since with it the plasma concentration of fentanyl can be adequately maintained at a steady level. However, when the patch is exposed to heat, it may lead to increased blood fentanyl levels that can cause dangerous respiratory depression $(3,4)$. We report a patient who had respiratory depression suspected of resulting from the use of a transdermal fentanyl patch while warming his upper body with a heater.

A 60-year-old man had pain and numbness in his back and legs secondary to lumbar canal stenosis at the L1-S1 level for which he had previously undergone laminotomy. Two years postoperatively, he had pain and numbness in his legs for which he was prescribed loxoprofen (180 mg orally), pregabalin (300 mg orally), oxycodone (15 mg orally) and rescue oxycodone (5 mg, 2 - 3 times a day). However, despite this medication, his visual analog scale (VAS) score for pain at rest was $82 \mathrm{~mm}$. He also suffered from constipation. Therefore, the $15 \mathrm{mg}$ oral oxycodone dose was changed to transdermal fentanyl (Durotep MT patch, JANSSEN, Japan; $2.1 \mathrm{mg}$ every 3 days). Due to inadequate pain relief with this dose, however, the dose of the transdermal fentanyl patch was increased from $2.1 \mathrm{mg}$ to $4.2 \mathrm{mg}$. This led to decreased pain in his legs and an improvement in VAS score for pain at rest to $55 \mathrm{~mm}$. The patient was also a known hypertensive for which he was being medicated with the calcium channel blocker cilnidipine (20 mg orally) for 2 years.

Three months after switching to transdermal fentanyl therapy, the patient complained of difficulty in breathing and respiratory depression when warming his upper body with a heater and in the bath. He visited the emergency department where he was diagnosed with respiratory depression associated with the fentanyl transdermal patch. Therefore, he was prescribed morphine sulfate (40 mg orally) instead of the transdermal fentanyl patch $(4.2 \mathrm{mg})$. With this, his respiratory depression decreased, but did not completely improve. Hence, suspecting that his difficulty in breathing was caused by myocardial ischemia, we recommended that he undergo coronary computed tomography, which led to a diagnosis of $90 \%$ stenosis of the distal left cir- cumflex coronary artery. Finally, the patient underwent percutaneous coronary intervention with a bare metal stent, which resulted in a decrease in his respiratory symptoms.

Transdermal fentanyl provides sustained analgesia for 72 hours; long-term treatment with transdermal fentanyl is generally well tolerated, particularly in view of the low incidence of potentially serious side effects, such as respiratory depression (5). However, when the patch is exposed to heat, it may lead to increased fentanyl blood levels that can cause dangerous respiratory depression $(3,4)$. Hence, in this case, since our patient complained of difficulty in breathing when warming himself with a heater or while in the bath, we believed that increased blood fentanyl levels were the cause of his respiratory depression. Furthermore, fentanyl is metabolized by P450 3A4, P450 3A4 inhibitors (e.g., ketoconazole, erythromycin and calcium blockers) and may lead to an increase in blood fentanyl concentration $(6,7)$. Our patient was being treated with calcium channel blockers. Hence, it is possible that the calcium blocker might have led to the increased fentanyl blood concentration and respiratory depression. Although discontinuation of fentanyl led to an improvement in our patient's respiratory depression, his symptoms did not completely disappear. As demonstrated by coronary computed tomography, his symptoms could also have resulted from myocardial ischemia. Thus, our patient's respiratory difficulties probably resulted from a combination of causes and mechanisms.

Yuka Matsuki, MD

Department of Anesthesiology \& Reanimatology,

Faculty of Medicine Sciences

University of Fukui

23-3 Matsuokashimoaizuki

Eiheiji-cho, Yoshida-gun

Fukui 910-1193, Japan

E-mail: ymatsuki@u-fukui.ac.jp

Maki Mizogami, MD

Department of Anesthesiology \& Reanimatology

Faculty of Medicine Sciences

University of Fukui

Fukui, Japan 
Mari Tabata, MD

Department of Anesthesiology \& Reanimatology

Faculty of Medicine Sciences

University of Fukui

Fukui, Japan

Yasunari Matsuki, MD

Department of Anesthesiology \& Reanimatology

Faculty of Medicine Sciences

University of Fukui

Fukui, Japan
Yoshikazu Yasuda, MD

Department of Anesthesiology \& Reanimatology

Faculty of Medicine Sciences

University of Fukui

Fukui, Japan

Kenji Shigemi, MD

Department of Anesthesiology \& Reanimatology

Faculty of Medicine Sciences

University of Fukui

Fukui, Japan

\section{References}

1. Mystakidou K, Parpa E, Tsilika E, Mavromati A, Smyrniotis V, Georgaki S, Vlahos L. Long-term management of non-cancer pain with transdermal therapeutic system-fentanyl. J Pain 2003; 4:298-306.

2. Manchikanti L, Vallejo R, Manchikanti KN, Benyamin RM, Datta S, Christo PJ. Effectiveness of long-term opioid therapy for chronic non-cancer pain. Pain Physician 2011; 14: E133-E156.

3. Frölich M, Giannotti A, Modell JH. Opi- oid overdose in a patient using a fentanyl patch during treatment with a warming blanket. Anesth Analg 2001; 93:647-648.

4. Newshan G. Heat-related toxicity with the fentanyl transdermal patch. J Pain Symptom Manage 1998; 16:277-278.

5. Milligan $\mathrm{K}$, Minet $\mathrm{ML}$, Borchert $\mathrm{K}$, Helmers H, Donald R, Kress HG, Adriaensen $H$, Moulin D, Järvimäki $V$, Haazen L. Evaluation of long-term efficacy and safety of transdermal fentanyl in the treatment of chronic noncancer pain. J Pain 2001; 2:197-204.

6. Trescot AM, Datta S, Lee M, Hansen $H$. Opioid pharmacology. Pain Physician 2008; 11:S133-S153.

7. Vallejo R, Barkin RL, Wang VC. Pharmacology of opioids in the treatment of chronic pain syndromes. Pain Physician 2011; 14:E343-E360.

\section{Heart Failure Associated with Pregabalin Use}

\section{TO THE EDITOR}

Pregabalin is a widely used antiepileptic drug that is also approved for the treatment of neuropathic pain. However, pregabalin may cause some adverse events, such as dizziness, somnolence, peripheral edema and weight gain (1). Moreover, we previously reported patients who developed muscle rigidity while taking oral pregabalin (2). Pregabalin has also been reported as causing deterioration of chronic heart failure (3-5). We report here a patient with no previous history of chronic heart failure who developed heart failure while on long-term oral pregabalin.

A 64-year-old male patient who had undergone rectal amputation 6 months previously presented with gradual onset of pain and paresthesia in his urethra after the operation. He had been implanted with a DDD pacemaker for complete atrioventricular block 2 years prior to his symptoms. His cardiothoracic ratio (CTR) on chest $\mathrm{x}$-ray was $52 \%$, with no evidence of pulmonary congestion. Echocardiography showed normal biventricular function and an estimated left ventricular ejection fraction of $68 \%$.

The patient was prescribed loxoprofen (180 mg orally), tramadol (100 $\mathrm{mg}$ orally), oxycodone (40 mg orally) and duloxetine (40 $\mathrm{mg}$ orally) for the pain. He was also prescribed pregabalin for the paresthesia, at a dose of $150 \mathrm{mg}$ orally, which is the recommended starting dose based on the value of Glomerular Filtration Rate (GFR) (his GFR at this time was $81.8 \mathrm{~mL} / \mathrm{min} / 1.73$ $\mathrm{m} 2$ ). A week later, the dose of pregabalin was increased to $300 \mathrm{mg} /$ day, which resulted in a decrease in the urethral pain and paresthesia.

Over a period of 3 months, however, the patient started developing peripheral edema, with the gradual appearance of facial edema and symptoms of volume overload, including a weight gain of $6 \mathrm{~kg}$ in one week. Hence, pregabalin was discontinued. Despite this, however, he was admitted to our hospital in a state of unconsciousness, with lip cyanosis, wheezing and orthopnea. At this time, his blood pressure was 124/66 $\mathrm{mmHg}$, heart rate was $100 \mathrm{bpm}$ and peripheral oxygen saturation was $80 \%$. Despite immediate administration of $100 \%$ oxygen via a face mask with the maintenance of spontaneous respiration, he continued to have difficulty breathing. Arterial blood gas analysis showed 
a $\mathrm{pH}$ of 7.271, arterial carbon dioxide tension of 68.9 $\mathrm{mmHg}$ and arterial oxygen tension ( $\mathrm{PaO} 2$ ) of 35.8 $\mathrm{mmHg}$. Chest X-ray demonstrated cardiomegaly (CTR $60 \%$ ) with a butterfly shadow, suggestive of congestive cardiac failure and pulmonary edema. The patient was administered $100 \%$ oxygen via a face mask at a flow rate of $10 \mathrm{~L} / \mathrm{min}$, and intravenous furosemide $20 \mathrm{mg} /$ day. With this therapy, he showed a good response by the next day. Over 7 days, he lost the weight he had acutely gained and there was an improvement in his peripheral and facial edema and heart failure.

Pregabalin is a calcium channel blocker that binds with high affinity to the alpha 2 delta subunits of voltage-gated calcium channels (6). Gong and colleagues (7) reported that human calcium channel alpha 2 delta mRNA subtypes have a high level of expression in the brain, heart and skeletal muscle. Murphy and colleagues (3) suggested a mechanism for heart failure induced by pregabalin. They hypothesized that pregabalin binds to the alpha 2 delta subunits of L-type calcium channels causing potassium-evoked attenuation of calcium ion influx, thus exerting a deleterious effect on myopathic ventricles. They also suggested that pregabalin directly promotes salt and water retention by the kidneys, which may also contribute to its potential to cause congestive heart failure (3). Our patient, who had normal biventricular function and a left ventricular ejection fraction of $68 \%$, although with a DDD pacemaker, had worsening of ventricular function after therapy with pregabalin. Further, pregabalin also possibly caused a deterioration of his kidney function, resulting in his GFR of $60.1 \mathrm{~mL}$ min/1.73 m2 3 months after taking pregabalin.

We describe the development of heart failure in a patient taking pregabalin. Although pregabalin-induced deterioration of cardiac function has been previously reported in patients with chronic heart failure, this is the report describing pregabalin-induced heart failure in a patient without a previous history of chronic heart failure.
Yuka Matsuki, M.D.

Department of Anesthesiology \& Reanimatology

Faculty of Medicine Sciences

University of Fukui

23-3 Matsuokashimoaizuki, Eiheiji-cho

Yoshida-gun, Fukui 910-1193, Japan

E-mail: ymatsuki@u-fukui.ac.jp

Mitsuhiro Morikawa, M.D.

First Department of Surgery

Faculty of Medicine

University of Fukui

Fukui, Japan

E-mail: mmitsu@u-fukui.ac.jp

Takefumi Nishimoto, M.D.

Department of Neuropsychiatry

Faculty of Medical Sciences

University of Fukui

Fukui, Japan

E-mail: takefumi@u-fukui.ac.jp

Maki Mizogami M.D.

Department of Anesthesiology \& Reanimatology, Faculty of Medicine Sciences

University of Fukui

Fukui, Japan

E-mail: makikai@u-fukui.ac.jp

Kenji Shigemi M.D.

Department of Anesthesiology \& Reanimatology

Faculty of Medicine Sciences

University of Fukui

Fukui, Japan

E-mail: kshigemi@u-fukui.ac.jp

\section{References}

1. Freynhagen R, Strojek K, Griesing T, Whalen E, Balkenohl M. Efficacy of pregabalin in neuropathic pain evaluated in a 12-week, randomized, double-blind, multicentre, placebo-controlled trial of flexible-and fixed-dose regimens. Pain 2005; 115:254-263.

2. Matsuki Y, Tabata M, Nobukawa Y, Sakai M, Yasuda Y, Mizogami M, Shigemi K. Muscle rigidity associated with pregabalin. Pain Physician 2012; 15: E5-6.

3. Murphy N, Mockler M, Ryder M, Ledwidge $\mathrm{M}$, McDonald K. Decompensation of chronic heart failure associated with pregabalin in patients with neuropathic pain. J Card Fail 2007; 13:227-229.

4. Page RL 2nd, Cantu M, Lindenfeld J, Hergott LJ, Lowes BD. Possible heart failure exacerbation associated with pregabalin: Case discussion and literature review. J Cardiovasc Med 2008; 9:922-925.

5. De Smedt $\mathrm{RH}$, Jaarsma T, van den Broek SA, Haaijer-Ruskamp FM. Decompensation of chronic heart failure associated with pregabalin in a 73-year-old patient with postherpetic neuralgia: Case report. $\mathrm{Br}$ J Clin Pharmacol 2008; 66:327-328.

6. Field MJ, Cox PJ, Stott E, Melrose H, Of- ford J, Su TZ, Bramwell S, Corradini L, England S, Winks J, Kinloch RA, Hendrich J, Dolphin AC, Webb T, Williams D. Identification of the alpha2-delta-1 subunit of voltage-dependent calcium channels as a molecular target for pain mediating the analgesic actions of pregabalin. Proc Natl Acad Sci 2006; 103:17537-17542.

7. Gong HC, Hang J, Kohler W, Li L, Su TZ. Tissue-specific expression and gabapentin-binding properties of calcium channel alphazdelta subunit subtypes. J Membr Biol 2001; 184:35-43. 